\title{
DETERMINAÇÃO DA ATIVIDADE ANTIMICROBIANA DO EXTRATO HIDROALCOÓLICO DA PLANTA PLECTRANTHUS ORNATUS CODD (BOLDO CHINÊS)
}

\author{
Lauana Aparecida SANTOS ${ }^{1}$ \\ Juliana da Silva MENEZES ${ }^{2}$ \\ Luciana Rosa Alves RUFINO ${ }^{3}$ \\ Nelma de Mello Silva OLIVEIRA ${ }^{4}$ \\ João Evangelista FIORINI ${ }^{5}$
}

\footnotetext{
${ }^{1}$ Acadêmica do Curso de Biomedicina, Universidade José do Rosário Vellano. email: lauanexsantos@hotmail.com

${ }^{2}$ Aluna de Mestrado em Ciência Animal, Universidade José do Rosário Vellano.

${ }^{3}$ Técnica de Laboratório da Universidade José do Rosário Vellano.

${ }^{4}$ Médica Veterinária, Professora da Universidade José do Rosário Vellano.

${ }^{5}$ Farmacêutico, Professor da Universidade José do Rosário Vellano.
}

\section{Recebido em: 31/03/2014 - Aprovado em: 30/06/2014 - Disponibilizado em: 30/07/2014}

RESUMO: A planta Plectranthus ornatus Codd é originária dos países do Mediterrâneo e Oriente Próximo. Na medicina popular é indicada para males do fígado e problemas da digestão. É utilizada no tratamento para o controle da gastrite, na dispepsia, azia, mal estar gástrico e ressaca. Este trabalho teve como objetivo a avaliação antimicrobiana do extrato de $P$. ornatus, utilizando-se 15 microrganismos padronizados. Os testes antimicrobianos foram realizados em ágar Mueller Hinton pela técnica de poços. Foram utilizados os testes de microdiluição em caldo, para a determinação da Concentração Inibitória Mínima (CIM) e semeadura, em placas contendo ágar Mueller Hinton para a Concentração Microbicida Mínima (CMM). Os testes de microdiluição em caldo demonstraram que o extrato nas concentrações de 20,31; 325 e 650 mg/mL inibiu o crescimento bacteriano de Bacillus cereus, Streptococcus pyogenes e Enterococcus faecalis, respectivamente. Para o fungo Saccharomyces cerevisiae os testes de microdiluição demonstraram inibição do crescimento na concentração de $1300 \mathrm{mg} / \mathrm{mL}$. Para as demais cepas testadas o extrato não demonstra atividade. Em decorrência da crescente resistência múltipla microbiana aos antibióticos as pesquisas para o desenvolvimento de novos medicamentos que sejam economicamente viáveis e com margem de segurança efetiva têm ganhado espaço na comunidade científica.

Palavras Chave: Plectranthus ornatus. Ação antimicrobiana. Concentração inibitória mínima. Concentração microbicida mínima. Produtos naturais.

\section{DETERMINATION OF THE PLANT EXTRACT HYDROALCOHOLIC PLECTRANTHUS ORNATUS CODD (BILBERRY CHINESE) ANTIMICROBIAL ACTIVITY}

\begin{abstract}
The Plectranthus ornatus Codd plant is originally from the Mediterranean and Near East. In folk medicine it is indicated for liver ailments and digestion problems. It is used in treatment to the control gastritis in dyspepsia, heartburn, upset stomach and hangover. This study aimed at the evaluation of antimicrobial extract of $P$. ornatus, using 15 standardized microorganisms. Tests were used broth microdilution for determining the Minimum Inhibitory Concentration (MIC) and sowing in plates containing Mueller Hinton agar for Microbicide Minimum Concentration (CMM). The broth microdilution tests showed that the concentrations of 20.31, 325 and $650 \mathrm{mg} / \mathrm{mL}$ inhibited bacterial growth of Bacillus cereus, Streptococcus pyogenes and Enterococcus faecalis, respectively. For yeast Saccharomyces cerevisiae microdilution the tests demonstrated growth inhibition at a concentration of $1300 \mathrm{mg} / \mathrm{mL}$. For all other strains tested extract shows no activity. Due to the increasing microbial resistance to multiple antibiotics the research or development new drugs that are economically viable and have effective safety margin.

Keywords: Plectranthus ornatus. Antimicrobial action. Minimum inhibitory concentration. Minimum microbicide concentration. Natural products
\end{abstract}




\section{Introdução}

O surgimento e a disseminação de microrganismos resistentes aos agentes antimicrobianos disponíveis no mercado têm sido relatados há décadas na literatura, incentivando a busca por novas fontes de substâncias com atividades antimicrobianas que sejam eficientes, como as plantas utilizadas na medicina popular (MENDES et al., 2011).

O tratamento de doenças utilizando-se extratos vegetais consiste em um dos métodos mais antigos da medicina natural. A origem deste conhecimento ainda é muito remota; acredita-se que grande parte foi adquirida por meio da observação do instinto humano e animal. Dessa maneira, o homem passou a distinguir plantas comestíveis, ou que poderiam tratar doenças, de plantas tóxicas. Este conhecimento foi transmitido de geração para geração pelas comunidades que conviviam com ervas e dependiam delas para tratar doenças (GONÇALVES et al., 2000).

O Brasil apresenta grande potencial para o desenvolvimento de estudos e de descobertas de plantas medicinais e de fármacos à base delas, pois cerca de $20 \%$ das 250 mil espécies medicinais catalogadas pela United Nations Educational Scientific and Cultural Organization (UNESCO), facilita o aproveitamento do potencial curativo dos vegetais para o tratamento de doenças no país
(DRUMOND et al., 2004). O ecossistema amazônico é detentor de uma das regiões de maior biodiversidade do planeta, apresenta inúmeras espécies vegetais com propriedades medicinais relatadas e outras em que seus efeitos terapêuticos ainda são desconhecidos (MENDES et al., 2011; BRASILEIRO et al.,2004).

Os boldos pertencem a um grupo de espécies de plantas com propriedades colagogas. O boldo do Chile (Peumus boldus Molina) é conhecido como verdadeiro boldo (MAURO et al., 2008). Brandão et al., (2006) registram na primeira Farmacopeia Brasileira, de 1929, que a planta popularmente conhecida como boldo era o Boldo do Chile; a diferenciação e correta identificação das espécies é importante, pois o mesmo e o falso boldo (Plectranthus barbatus Ard.) possuem compostos que causam efeitos colaterais.

A família Lamiaceae é originária principalmente de países do Mediterrâneo e Oriente, consistindo de cerca de 200 gêneros e 3200 espécies. Dentre os gêneros, destacamse o Plectranthus, que inclui diversos representantes na utilização terapêutica (DUARTE e LOPES, 2005). As espécies de Plectrathus são ricas em diterpenos e são usadas na medicina popular em várias partes do mundo (ALBUQUERQUE et al., 2003; ALBUQUERQUE et al., 2003). 
A planta Plectranthus ornatus Codd é popularmente conhecida como boldo chinês, boldo gambá, boldo miúdo ou boldo rasteiro (MAURO et al., 2008). Na medicina popular é indicada para males do fígado e problemas da digestão. Pode ser também utilizada no tratamento para controle da gastrite, na dispepsia, azia e mal estar gástrico, o seu sabor amargo é estimulante da digestão e do apetite (CODD, 1985; MAURO et al., 2008).

As folhas contêm substâncias que apresentam atividades analgésicas, não apresentando efeito colateral. Foram observadas leve atividade sedativa, que pode estar associada à ação analgésica, bem como bactericida e fungicida, ainda não especificadas pela literatura (MAURO et al., 2008).

\section{Materiais e métodos}

2.1 Coleta das espécimes

As folhas de Plectranthus ornatos foram coletas no município de Alfenas, estado de Minas Gerais, Brasil, à beira da Rodovia MG - 179, Km 0, em setembro de 2011 e identificadas pela equipe do Laboratório de Botânica da Universidade Federal de Alfenas (UNIFAL). A exsicata do vegetal está armazenada no Herbário da Universidade José do Rosário Vellano (UNIFENAS), Alfenas, sob o n 320 .

\subsection{Obtenção do extrato}

O extrato das folhas frescas de Plectranthus ornatos foi obtido utilizando-se, como agente extrator, álcool etílico a $70 \%$, conforme técnica descrita por Caceres et al., (1995) e Farmacopeia Brasileira $5^{\mathrm{a}}$ edição (2010). Foram pesados $400 \mathrm{~g}$ das folhas da planta e colocados em $1600 \mathrm{~mL}$ de álcool a $70 \%$. Essa mistura foi macerada em balão volumétrico $(2000 \mathrm{~mL})$ e armazenada à temperatura ambiente por 15 dias, ao abrigo da luz. Após, a mesma foi filtrada e mantida sob refrigeração a $4^{\circ} \mathrm{C}$ em frasco âmbar estéril. Posteriormente, foi concentrada em evaporador rotatório e liofilizada. Na hora do uso, a mesma foi ressuspensa em água destilada estéril e passada em filtro Millipore ${ }^{\circledR}(0,22 \mu \mathrm{m})$.

\subsection{Ação antimicrobiana}

Os ensaios de ação antimicrobiana foram realizados com bactérias e fungos padronizadas (ATCC e NEWPROV). As bactérias foram Bacillus cereus (ATCC 11778), Bacillus stearothermophilus (ATCC 7953), Bacillus subtilis (ATCC 6633), Enterobacter aerogenes (ATCC 13048), Enterococcus faecalis (ATCC 29212), Escherichia coli (ATCC 25922), Klebsiella pneumoniae (ATCC 13883), Proteus mirabilis (ATCC 25933), Pseudomonas aeruginosa (ATCC 25619), Shigella flexineri (NEWPROV 0122), Salmonella typhimurium (ATCC 14028), Staphylococcus aureus (NEWPROV 25923), Streptococcus pyogenes 
(NEWPROV 19615). Para fungos utilizou-se Candida albicans (ATCC 1023-1) e Saccharomyces cerevisiae (ATCC 2601). As amostras de bactérias foram mantidas em ágar BHI e os fungos, em ágar Sabouraud, à temperatura de $4^{\circ} \mathrm{C}$, até o momento de realização dos testes.

\subsection{Avaliação do perfil de sensibilidade}

A atividade antimicrobiana do extrato vegetal de Plectranthus ornatos foi avaliada por meio do teste de difusão em ágar, Concentração Inibitória Mínima (CIM) e Concentração Microbicida Mínima (CMM), de acordo com os padrões do National Committee for Clinical Laboratory Standards (NCCLS, 2002) ${ }^{15}$. Foram utilizadas as concentrações de 1300; 650; 325; 162,5; 81,$25 ; 40,62 ; 20,31 ; 10,15 ; 5,07 ; 2,53$ $\mathrm{mg} / \mathrm{mL}$. As determinações do perfil de sensibilidade foram realizadas de acordo com a metodologia de microdiluição em meio RPMI 1640 para leveduras, conforme protocolo M27A3 descrito pela CLSI, (2008) e microdiluição em caldo Mueller Hinton para bactérias, conforme protocolo M7A6 descrito pela CLSI, (2003). Os ensaios foram realizados em duplicata.

A CIM foi determinada nos extratos que apresentaram atividade inibitória no teste de difusão em ágar segundo NCCLS, (2002) em microplacas, nas quais foram colocados 150 $\mu \mathrm{L}$ do caldo Mueller Hinton concentrado (2x) em todos os poços. No primeiro poço foi adicionado o extrato com concentração de $1300 \mathrm{mg} / \mathrm{mL}$, e a partir deste foram preparadas diluições seriadas decrescentes. Foram inoculados nos poços $10 \mu \mathrm{L}$ da suspensão microbiana com turvação equivalente ao tubo de 0,5 da Escala de MacFarland.

A CMM foi realizada nas concentrações do extrato que apresentaram inibição para o crescimento bacteriano. A confirmação da ação bacteriostática/bactericida e/ou fungistática/fungicida das diluições foi realizada através do plaqueamento da diluição específica, e das concentrações imediatamente superior e inferior no meio de cultura ágar Mueller Hinton. Após, as placas foram analisadas com observação da presença ou ausência de crescimento microbiano.

Estatística - para os ensaios de ação antimicrobiana foi adotada estatística descritiva.

\section{Resultado}

$\mathrm{Na}$ verificação da atividade antimicrobiana do extrato de $P$. ornatus, observou-se formação de halos de inibição entre 18 a $22 \mathrm{~mm}$ sobre o crescimento de bactérias Gram-positivas Bacillus cereus ATCC 11778, Enterococcus faecalis ATCC 29212 e Streptococcus pyogenes NEWPROV 19615 e sobre o crescimento do fungo Saccharomyces cerevisiae ATCC 2601. Não houve formação de halos para os seguintes 
microrganismos: Bacillus subtilis ATCC 6633, Bacillus sterothermophilus ATCC 7953, Candida albicans NEWPROV 0031, Enterobacter aerogenes ATCC 13046, Escherichia coli ATCC 25922, Klebsiella pneumoniae ATCC 13883, Proteus mirabilis ATCC 25933, Pseudomonas aeruginosa ATCC 25619, Staphylococcus aureus NEWPROV 25923, Streptococcus pyogenes NEWPROV 19615, Shigella flexineri NEW

PROV 0122, Salmonella typhimurium NEWPROV 14028 (Tabela 1).
Os resultados obtidos, no que diz respeito à Concentração Inibitória Mínima (CIM) e Concentração Microbicida Mínima (CMM), foram positivos para as cepas de Bacillus cereus (ATCC 11778) Enterococcus faecalis (ATCC 29212) na concentração de $20,31 \mathrm{mg} / \mathrm{mL}$ e Streptococcus pyogenes (NEWPROV 19615) na concentração de 325 $\mathrm{mg} / \mathrm{mL}$, para o fungo Saccharomyces cerevisiae (ATCC 2601) foi observada apenas a Concentração Inibitória Mínima (CIM) de $1300 \mathrm{mg} / \mathrm{mL}$, a mesma para a Concentração Microbicida Mínima (CMM) (Tabela 1).

Tabela 1 - Halos de Inibição, Concentração Inibitória Mínima e Concentração Microbicida Mínima do extrato hidroalcoólico de $P$. ornatus

\begin{tabular}{lccc}
\hline \multicolumn{1}{c}{ MICRORGANISMOS } & $\begin{array}{c}\text { Halos de Inibição } \\
\mathbf{m m}\end{array}$ & $\begin{array}{c}\text { CIM } \\
\mathbf{m g} / \mathbf{m L}\end{array}$ & $\begin{array}{c}\text { CMM** } \\
\text { mg/mL }\end{array}$ \\
\hline Bacillus cereus (ATCC 11778) & 18 & 20,31 & 1300,0 \\
Bacillus subtilis (ATCC 6633) & - & - & - \\
Bacillus stearothermophilus (ATCC 7953) & - & - & - \\
Candida albicans (ATCC 10231) & - & - & - \\
Enterobacter aerogenes (ATCC 13046) & - & - & - \\
Enterococcus faecalis (ATCC 29212) & 22 & 20,31 & 1300,0 \\
Escherichia coli (ATCC 25922) & - & - & - \\
Klebsiella pneumoniae (ATCC 13883) & - & - & - \\
Proteus mirabilis (ATCC 25933) & - & - & - \\
Pseudomonas aeruginosa (ATCC 25619) & - & - & - \\
Saccharomyces cerevisiae (ATCC 2601) & 29 & 1300,0 & - \\
Shigella flexineri (NEWPROV 0122) & - & - & - \\
Salmonella typhimurium (NEWPROV 14028) & - & - & - \\
Staphylococcus aureus (ATCC 6538) & - & - & 1300,0 \\
Streptococcus pyogenes (NEWPROV 19615) & 22 & 325,0 & \\
\hline
\end{tabular}

Fonte: $\mathrm{O}$ autor

Nota: * Concentração Inibitória Mínima

**Concentração Microbicida Mínima 


\section{Discussão}

Fernandes et al., (2011) verificaram que a atividade antimicrobiana de extratos e óleos vegetais, deve-se aos produtos do metabolismo secundário, como terpenoides e compostos fenólicos, sendo eles flavonoides e saponinas, que em sua forma pura também exibem ação antimicrobiana. A diferença dos achados de atividade antimicrobiana descritos na literatura sobre plantas pode estar relacionada com a quantidade de cada princípio ativo presente nos extratos, o uso de técnicas e procedimentos diferentes, bem como a época do ano em que foi feita a colheita do material (FERNANDES et al., 2011).

Brasileiro et al., (2006) avaliaram a atividade antimicrobiana e citotóxica do extrato de $P$. ornatus e outras 31 espécies de empregando o método de difusão em ágar e o teste de letalidade de Artemia, observando que, dentre as cepas testadas, não houve inibição do crescimento de Sthapylococcus aureus e Escherichia coli, confirmando os resultados obtidos nesta pesquisa, demostrando também que o extrato apresentou toxicidade às larvas de Artemia salina (DL50<1000 ppm).

Ao realizarem estudos com plantas medicinais, Nogueira; Diniz e Lima, (2008) observaram que cepas de $S$. aureus foram sensíveis na presença do extrato de Plectranthus amboinicus. Na investigação utilizaram 10 isolados de $S$. aureus, 8 de Pseudomonas aeruginosa, 4 de Candida albicans e 4 de Candida krusei, isolados de otite externa aguda. Pseudomonas aeruginosa foi resistente a todos os extratos de plantas testados.

Lopes e Almeida, (2011) também avaliaram a atividade antibacteriana do extrato da fruta de Morinda citrifolia L., sobre as cepas de $S$. aureus e $E$. coli, demonstrando que $\mathrm{o}$ extrato também não inibiu $\mathrm{o}$ crescimento das mesmas.

Pinho et al., (2012) realizaram estudo sobre atividade antimicrobiana utilizando os extratos das plantas aroeira, barbatimão e erva baleeira em ensaios de difusão em ágar sobre as cepas de $S$. aureus e E. coli. Foi demonstrado que os extratos das plantas em estudo inibiram o crescimento somente de $S$. aureus, mas não de E. coli.

Em ensaio realizados com os extratos alcoólicos de própolis, com concentração de 11 e $20 \%$, ficou demonstrada a inibição do crescimento de Bacillus cereus, Bacillus subtilis, Klebisiela pneumoniae, Streptococcus pyogenes, Enterobacter aerogenes, Micrococcus luteus, Candida albicans e Saccharomyces cerevisae (JOSÉ et al., 2008).

Silva et al., (2012) avaliaram a atividade antioxidante e antimicrobiana do extrato de Mimosa caesalpiniifolia Benth, nas cepas de Bacillus cereus, Candida albicans, Candida krusei, Candida glabrata, Candida 
parapsilosis, Candida tropicalis, Escherichia coli, Pseudomonas aeruginosa e Staphylococcus aureus, utilizando a técnica de difusão em ágar. Constataram que o extrato exibiu atividade inibitória de crescimento para todos os microrganismos testados.

Lopes et al., (2006) realizaram estudo avaliando a atividade antimicrobiana do extrato seco de insulina e óleo de copaíba em cepas bacterianas e fúngicas (Salmonella typhimurium, Staphylococcus aureus, Staphylococcus epidermidis, Enterococcus faecalis, Escherichia coli, Bacillus cereus, Bacillus subtilis, Candida albicans e Cryptococcus neoformans). Verificaram que o óleo de copaíba inibiu o crescimento de $S$. aureus, E. coli , B. subtilis e E. faecalis, sendo os demais microrganismos, resistentes. A CIM para os microrganismos sensíveis variou de 6,05 a 30,25 mg/mL, com CMM de $18,15 \mathrm{mg} / \mathrm{mL}$ para E. faecalis. O extrato de insulina não demostrou ação antimicrobiana.

Os extratos de orégano, tomilho, lipia, gengibre, sálvia, alecrim e manjericão foram estudadas por Pozzo et al., (2011). Os ensaios de ação antimicrobiana sobre 32 cepas de $S$. aureus isolados de mastite bovina demonstraram ação inibitória em todos os microrganismos testados.

A investigação da atividade antimicrobiana e antioxidante da planta Ziziphus joazeiro, conhecida como juazeiro ou laranjeira do vaqueiro, utilizando-se a técnica de difusão em ágar e Concentração Inibitória Mínima, evidenciou que o extrato das folhas e cascas possuíam atividade antioxidante e antimicrobiana em $70 \%$ das cepas analisados destacando-se Candida albicans, Enterococcus faecalis, Escherichia coli, Pseudomonas aeruginosa e Streptococcus pyogenes. O extrato das folhas mostrou CIM entre $0,25-0,5 \mathrm{mg} / \mathrm{mL}$ contra Micrococcus luteus e entre 0,125-0,250 $\mathrm{mg} / \mathrm{mL}$ contra Mycobacterium smegmatis, enquanto o extrato da casca apresentou CIM entre $0,5-1,0 \mathrm{mg} / \mathrm{mL}$ para $M$. smegmatis (SILVA et al., 2011).

Estudos fitoquímicos possibilitaram o isolamento de diterpenos do gênero Plectranthus, sendo a ornantina A, barbatusina, labdano e forskolina os princípios ativos do gênero Plectranthus sp. Identificou-se também a presença de alquilfenois e flavonoides (OLIVEIRA et al., 2005; WANNMACHER, 2002). Tais princípios ativos poderiam estar relacionados com a ação antimicrobiana observada nos resultados aqui descritos.

Devido à grande variedade da composição química de plantas, estudos realizados detectaram a presença de flavonoides, sendo estes possuidores de várias ações farmacológicas, destacando-se a ação anti-inflamatória, cicatrizante, antitumoral, antimicrobiana e principalmente antifúngica (MENDES et al., 2011; GONÇALVES et al., 2000; SILVA et al., 2012; WANNMACHER, 
2002; BRUNETON, 1999; DOTTO et al., 2006 e OLIVEIRA et al.,2005). No presente estudo, observou-se a atividade antifúngica do produto avaliado em diversas concentrações, obtendo-se a inibição do fungo Saccharomyces cerevisae em presença do extrato de $P$. ornatus. No entanto, não houve inibição de Candida albicans, pressupondo-se a interferência de vários fatores.

Os flavonoides e taninos apresentam a habilidade de inativar enzimas e complexarem-se com proteínas extracelulares, proteínas solúveis e com a parede celular das bactérias, configurando os prováveis mecanismos de ação antimicrobiana. A total ruptura de membranas microbianas pode ser dada por flavonoides de caráter lipofílico (MENDES et al., 2011; GONÇALVES et al., 2000 e SILVA et al., 2012). Sendo assim, é possível que esses compostos estejam presentes no extrato de $P$. ornatos, sendo responsáveis por sua atividade antimicrobiana.

Em estudos de atividade antimicrobiana "in vitro" utilizando-se extratos brutos de plantas, o potencial antimicrobiano muitas vezes não está relacionado a um único princípio ativo. Portanto, o processo de isolamento de substâncias ativas que estejam presentes nos extratos em estudo pode inviabilizar a utilização da planta como fitoterápico, pois sua ação está ligada à associação de vários princípios ativos e não a um único que esteja presente (ANDRADE E FIORINI, 2009).

\section{Conclusão}

O extrato hidroalcoólico das folhas de P. ornatus apresentou Concentração Inibitória Mínima (CIM) de 20,31 mg/mL até 1300 $\mathrm{mg} / \mathrm{mL}$ variando com a espécie testada. A Concentração Bactericida Mínima (CMM) foi constatada em três dentre as quatro cepas bacterianas que apresentaram halos de inibição. Este estudo reafirma a importância de investigações etnofarmacológicas na seleção de plantas com indicação terapêutica.

\section{Agradecimentos}

Ao CNPq, pela concessão da bolsa de Iniciação Científica.

\section{Referências}

ALBUQUERQUE, R.L.; MACHADO, M.I.L.; SILVA, M.G.V.; MORAIS, S.M.; MATOS, F.J.A.; LIMA, L.B. Novo diterpeno isolado das folhas de Plectranthus ornatus, Reunião Anual da Sociedade Brasileira de Química, maio de 2003.

ALBUQUERQUE, R.L.; MACHADO, M.I.L.; SILVA, M.G.V.; MORAIS, S.M.; MATOS, F.J.A.; LIMA, L.B. Estudo químico e Atividade do óleo essencial de Plectranthus grandis Will e Plectranthus ornatus Codd. Reunião Anual da Sociedade Brasileira de Química, maio de 2003. 
ANDRADE, J.S.; FIORINI, J.E. Atividade

Antimicrobina "in vitro", Ação cicatrizante e anti-inflamatória "in vivo" do extrato de Luffa opercolata. [Tese 58-65] Alfenas,

Universidade José do Rosário Vellano; 2009.

BRANDÃO, M.G.L.; COSENZA, G.P.;

MOREIRA, R.A.; MONTE-MOR, R.L.M.

Medicinal plants and other botanical products

from the Brazilian Official

Pharmacopoeia. Rev Bras Farmacogn. 2006;

16:408-420.

BRASILEIRO, B.G.; PIZZIOLO, V.R;

RASLAN, D.S.; JAMAL, C.M.; SILVEIRA,

D. Antimicrobial and cytotoxic activities

screening of some Brazilian medicinal plants

used in Governador Valadares district. Rev

Bras Ciên Farm. 2006; vol. 42, n. 2, abr./jun.

BRUNETON, J. Pharmacognosy:

phytochemistry medicinal plants. 2. ed. Paris:

Lauvoisier, 1999; 1119 p.

CARCERES, A.; MENÉNDEZA, H.;

MÉDEZ, E. et al. Antigonorrhoel activity of plntas used in Guatemala for the treatment of sexually transmitted diseases. $\mathbf{J}$

Etnopharmacol. 1995; 48(2):85-8.

Clinical and Laboratory Standards Institute (CLSI). Methods for dilution antimicrobial susceptibility tests for bacteria that grow aerobically. Approved standard. 6th ed. M7A6. Wayne, PA: CLSI; 2003.
Clinical and Laboratory Standards Institute (CLSI). Reference method for broth dilution antifungal susceptibility testing of yeasts. Approved Standard-Third Edition. M27-A3. Wayne, PA: CLSI; 2008.

CODD, L.E. Flora of Southern Africa;

Botanical Research Institute, Department of Agriculture and Water Supply : Pretoria. 1985; v.28, Part 4, p.137-151.

DOTTO, S.R.; TRAVASSOS, R.M.C.; FERREIRA, R.; SANTOS, R.; WAGNER, M. Avaliação da ação antimicrobiana de medicações usadas em endodontia. Rev Odonto Ciência. 2006; v.21, n.5.

DRUMOND, M.R.S.; CASTRO, R.D.; ALMEIDA, R.V.D.; PEREIRA, M.S.V.;

PADILHA, W.W.N. Estudo comparativo in vitro da atividade antibacteriana de produtos fitoterápicos sobre bactérias cariogênicas. Pesq Bras Odontoped Clin Integr. 2004; 4(1): 33-8.

DUARTE, M.R e LOPES, J.F.

Morfoanatomia foliar e caulinar de Leonurus sibiricus, Lamiaceae, Rev Acta Farm

Bonaerense. 2005; vol. 24, n 1.

FARMACOPEIA BRASILEIRA. 5th ed. São

Paulo: Atheneu; 2010. v. 1-2.

FERNANDES, A.P.; RIBEIRO, G.E.;

RUFINO, L.R.A.; SILVA, L.M.;

BORIOLLO, M.F.G.; OLIVEIRA, N.M.S.;

FIORINI, J.E. Efeito do extrato 
hidroalcoólico de Pyrostegia venusta na

mutagênese "in vivo", e avaliação

antimicrobiana, e interferência no crescimento

e diferenciação celular “in vitro". Rev Med

Minas Gerais. 2011; 21(3):272-279.

GONCALVES et al. A cura está na natureza:

Medicina natural. São Paulo: Editora Brasil.

2000; 21 p. 544.

JOSÉ, T.D.S.; ASSUNPÇÃO, R.;

OLIVEIRA, N.M.S.; FIORINI, J.E. Análise

da atividade antimicrobiana com diferentes

extratos de própolis In: I Congresso de

Biomedicina. Alfenas, Universidade José do

Rosário Vellano (UNIFENAS); 2008.

LOPES, K.C.; PERREIRA, M.A.;

NASCIMENTO, L.C.; FIORINI, J.E.

Avaliação da atividade antimicrobiana e ação de Cissus sicynoides e Copaifera langsdorffii

na diferenciação se sistemas eucarióticos

unicelulares. In: Seminário de Iniciação

Cientifica. Alfenas, Universidade José do

Rosário Vellano (UNIFENAS); 2006.

LOPES, L.C.; ALMEIDA, J.V.P. Atividade

antibacteriana do extrato hidroalcoólico da

fruta Morinda citrifolia L. (NONI) em cepas

de S. aureus e E. coli. Rev Hig Ali. 2011; v. 25, p. 162-168.

MAURO, C.; SILVA, C.P.; MISSIMA, J.;

OHNUKI, T.; RINALDI, R.B.; FROTA, M.

Estudo anatômico comparado de órgãos vegetativos de boldo miúdo, Plectranthus
ornatusCodd. e malvariço, Plectranthus

amboinicus(Lour.) Spreng. - Lamiaceae. Rev. bras. farmacogn. 2008; 18(4): 608-613.

MENDES, L.P.M.; MACIEL, K.M.; VIEIRA, A.B.R.; MENDONÇA, L.C.V.; SILVA, R.M.F.; ROLIM-NETO, P.J.; BARBOSA, W.L.R.; VIEIRA, J.M.S. Atividade antimicrobiana de extratos etanólicos de Peperomia pellucida e Portulaca pilosa. Rev Ciên Farm Básica Apl. 2011; 32(1): 121-5.

(NCCLS) National Comitte for Clinical Laboratory Standart. Padrões de desempenho para teste de susceptibilidade antimicrobiana: padrão M2-A6 aprovado, $6^{\text {a }}$ ed. Wayne, PA: NCCLS; 2002.

NOGUEIRA, J.C.R.; DINIZ, M.F.M.; LIMA, E.O. Atividade antimicrobiana in vitro de produtos vegetais em otite externa aguda. Rev. Bras. Otorrinolaringol. 2008;74(1):11824.

OLIVEIRA, A.C.P.; ENDRINGER, D.C.; AMORIM, L.A.S.; BRANDÃO, M.G.L.; COELHO, M.M. Effect of the extracts and fractions of Baccharis trimera and Syzygium cumini on glycaemia of diabetic and nondiabetic mice. J Ethnopharmacol. 2005; v. 16, p. 465-469,.

OLIVEIRA, P.M.; FERREIRA, A.A.;

SILVEIRA, D.; ALVES, R.B.;

RODRIGUES, G.V.; EMERENCIANO, V.P.; RASLAN, D.S. Diterpenoids from the Aerial 
Parts of Plectranthus ornatus. J Nat Prod.

2005; Vol. 68.

PINHO, L.; SOUZA, P.N.S.; SOBRINHO, E.M.; ALMEIDA, A.C.; MARTINS, E.R.

Atividade antimicrobiana de extratos

hidroalcoolico das folhas de alecrim-pimenta, aroeira, barbatimão, erva baleeira e do farelo da casca de pequi. Rev Ciência Rural. 2012; v.42, n.2, p.326-331.

POZZO, M.D.; SANTURIO, D.F.;

ROSSATTO, A.C.;VARGAS, S.H.; ALVES, E.S.; LORETO, J.V. Activity of essencial oils from spices Staphylococcus spp. Isolated from bovine mastites. Arq Bras Med Vet Zootec. 2011; v.63, n.5, p.1229-1232. SILVA, M.J.D.; ENDO, L.H.; DIAS, A.L.T.; SILVA, G.A.; SANTOS, M.H.; SILVA, M.A.
Avaliação da atividade antioxidante e antimicrobiana dos extratos e frações orgânicas de Mimosa caesalpiniifolia Benth. (Mimosaceae). Rev Ciên Farm Básica Apl. 2012; 33(2):267-274.

SILVA, T.C.L.; ALMEIDA, C.C.B.R.; VERAS, F. J.; PEIXOTO, S.T.J.S.; AMORIM, E.L.C.; COSTA, E.P.; ARAÚJO, J.M. Atvidades antioxidante e antimicrobiana de Ziziphus joareiro mart. (Rhamnaceae): avaliação compartiva entre cascas e folhas. Rev. Ciên Farm Básica Apl. 2011; 32(2):193199.

WANNMACHER, L. Uso indiscriminado de antibióticos e resistência microbiana: uma guerra perdida? Boletim de Saúde. 2002; v. 23, n. 12 , p. 1127-1141. 